\title{
Robust Synchronization of Class Chaotic Systems Using Novel Time-Varying Gain Disturbance Observer-Based Sliding Mode Control
}

\author{
Yang Wang $\mathbb{D}$, Zhen Wang $\mathbb{D}$, and Lingyun Kong $\mathbb{( i t}$ \\ School of Science, Xijing University, Xi'an, China \\ Correspondence should be addressed to Yang Wang; 2013100280@mail.nwpu.edu.cn
}

Received 16 August 2020; Revised 19 September 2020; Accepted 2 April 2021; Published 21 April 2021

Academic Editor: Hassan Zargarzadeh

Copyright (c) 2021 Yang Wang et al. This is an open access article distributed under the Creative Commons Attribution License, which permits unrestricted use, distribution, and reproduction in any medium, provided the original work is properly cited.

\begin{abstract}
For synchronization of a class of chaotic systems in the presence of nonvanishing uncertainties, a novel time-varying gain observer-based sliding mode control is proposed. First, a novel time-varying gain disturbance observer (TVGDO) is developed to estimate the uncertainties. Then, by using the output of TVGDO to modify sliding mode control (SMC), a new TVGDO-based SMC scheme is developed. Although the observation and control precision of conventional fixed gain disturbance observer-based control (FGDOC) for chaotic systems can be guaranteed by a high observer gain, the undesirable spike problem may be caused by the high gain if the initial values of estimate and true states are not equal. The most attractive feature of this work is that the newly proposed TVGDO can eliminate the spike problem by developing a time-varying gain scheme. Finally, the effectiveness of the proposed method is demonstrated by the numerical simulation.
\end{abstract}

\section{Introduction}

In the past decades, with the development of theoretical analysis methods of chaos, many chaos systems such as the Lorenz system [1], Rossler system [2], and Chen system [3] have been wildly studied. These theoretical advancements of chaotic systems have been influentially applicated in many fields, such as power electrical systems [4, 5], robotics [6], lasers [7], and secure communications [8]. Among these applications, to achieve the desired chaotic characteristic, the high-precision synchronization problem is the key problem that must be solved. The objective of synchronization control between the master and slave chaotic systems can be achieved when the instantaneous states of the two systems become identical. Note that unmodeling dynamic, environmental disturbance, and uncertainty parameters usually exist in the slave chaotic systems [9]. These uncertainties can greatly affect the synchronization performance. To improve the robustness of chaos synchronization in allusion to uncertainties, many modern robust control theories have been applied to design synchronization controllers, including $H_{\infty}$ robust control $[10,11]$, adaptive control $[12,13]$, neural network control $[14,15]$, observerbased control $[16,17]$, and sliding mode control (SMC) [18-22]. Among these schemes in [10-22], due to its advantage of low sensitivity to uncertainties and fast dynamic response, SMC is a good candidate to achieve high-precision synchronization in the presence of uncertainties. In $[18,19]$, the authors adopted the linear sliding mode surface to design a synchronization controller for chaotic systems. In [20, 21], the terminal sliding mode method was investigated to guarantee the fast finite-time synchronization of uncertain chaotic systems. In [22], to establish invariance of the system with uncertainties from the initial time instant, the integral sliding mode control scheme was investigated to design the synchronization controller. However, the robustness of these conventional SMC schemes in [18-22] is guaranteed by using the discontinuous control terms. The discontinuous terms can bring an undesirable chattering problem.

It is well known that the chattering problem may affect the synchronization precision and cause the instability of the closed-loop system [23-25]. Thus, research on chattering- 
free SMC synchronization scheme has the important practical and theoretical significance. Based on observer techniques, the chattering problem of conventional SMC can be eliminated by using the estimation of uncertainties to replace the discontinuous control terms of SMC. In [26, 27], the authors adopted the high-order sliding mode (HOSM) observers to estimate the uncertainties of the chaotic system. However, the HOSM observer used in $[26,27]$ must know the upper bound of uncertainties in advance. Since the characteristics of uncertainties are complex, it is difficult to know the upper bound. In [28-30], the SMC schemes were proposed by employing the disturbance observer (DO) to estimate the uncertainties in chaotic systems. In [31], the authors adopted the estimation of extended state observer (ESO) to modify the conventional SMC. Unlike HOSM observer in [26, 27], the DO and ESO in [28-31] does not require the upper bound of uncertainties.

For the DO-based and ESO-based SMC schemes in [28-31], to guarantee the observation and control precision, the observer gains of DO and ESO should be chosen as the high gains. However, the undesirable spike problem can be caused by the high observer gains when the initial values of estimate and true states are not equal. The spike problem may cause the saturation of control input and even the instability. Actually, it is difficult to obtain the initial values of state in advance for the most of practical systems. Thus, the DO-based and ESO-based SMC schemes in [28-31] may not work well in many practical situations.

In this study, a new time-varying gain disturbance observer (TVGDO) is proposed to estimate the lumped uncertainties of the chaotic system. And then, a novel TVGDObased SMC scheme is designed based on the output of TVGDO. The main contributions of this study lie in the following aspects:

(1) Compared with conventional DO and ESO used in the uncertain chaotic systems, the most attractive feature of the proposed TVGDO is that the spike problem can be eliminated on the condition of the initial values of estimate and true states are not equal.

(2) The proposed TVGDO-based SMC is spike-free and chattering-free. And the TVGDO-based SMC can guarantee the synchronization without using the upper bound information of uncertainties.

The remaining parts of this study are as follows. In Section 2, the synchronization control model, the design objective, and the motivation of this study are expounded. The main results are presented in Section 3. In Section 3.1, a novel observer TVGDO is developed, and the stability proof of TVGDO is presented. In Section 3.2, the spike-free characteristic of TVGDO is analyzed. In Section 3.3, a TVGDO-based SMC scheme is proposed, and the stability of the proposed controller is also obtained. In Section 4, a simulation verifies the effectiveness of both TVGDO and the proposed TVGDO-based SMC. In Section 5, the conclusion of the whole study is presented.
Notations. The following notations will be used in this study: $t$ denotes the time and the initial time is 0 . Let $\|\cdot\|$ denote the Euclidean norm of a vector and its induced norm of a matrix.

\section{Problem Formulation}

2.1. System Description. In this study, the dynamic of the master chaotic system is described as follows [12]:

$$
\left\{\begin{array}{l}
\dot{x}_{m 1}=f_{m 1}\left(\mathbf{X}_{m}, t\right), \\
\dot{x}_{m 2}=f_{m 2}\left(\mathbf{X}_{m}, t\right), \\
\vdots \\
\dot{x}_{m n}=f_{m n}\left(\mathbf{X}_{m}, t\right),
\end{array}\right.
$$

where $x_{m i}(i=1,2, \ldots, n)$ represents the states of the master system, $X_{m}=\left[\begin{array}{llll}x_{m 1} & x_{m 2} & \ldots & x_{m n}\end{array}\right]^{T}$ is the state vector, and $f_{m i}\left(X_{m}, t\right),(i=1,2, \ldots, n)$ is the nonlinear function and determines the chaotic characteristic.

The slave chaotic system is given as follows [12]:

$$
\left\{\begin{array}{l}
\dot{x}_{s 1}=f_{s 1}\left(\mathbf{X}_{s}, t\right)+\Delta f_{s 1}\left(\mathbf{X}_{s}, t\right)+u_{1}(t)+d_{1}(t), \\
\dot{x}_{s 2}=f_{s 2}\left(\mathbf{X}_{s}, t\right)+\Delta f_{s 2}\left(\mathbf{X}_{s}, t\right)+u_{2}(t)+d_{2}(t), \\
\vdots \\
\dot{x}_{s n}=f_{s n}\left(\mathbf{X}_{s}, t\right)+\Delta f_{s n}\left(\mathbf{X}_{s}, t\right)+u_{n}(t)+d_{n}(t),
\end{array}\right.
$$

where $x_{s i}(i=1,2, \ldots, n)$ represents the states of the slave system, $\quad \mathbf{X}_{s}=\left[\begin{array}{llll}x_{s 1} & x_{s 2} & \ldots & x_{s n}\end{array}\right]^{T}$ is the state vector, $f_{s i}\left(\mathbf{X}_{s}, t\right)(i=1,2, \ldots, n)$ is the nonlinear function, $u_{i}(t)(i=$ $1,2, \ldots, n)$ is the control input, and $\Delta f_{s i}\left(\mathbf{X}_{s}, t\right)$ and $d_{i}(t)(i=$ $1,2, \ldots, n)$ are the bounded uncertainty and disturbance, respectively. Like the conventional SMC, the uncertainties considered in this study are matched uncertainties, which imply that the uncertainties and control inputs exist in the same channel. It is assumed that all states of systems (1) and (2) are measured and noise-free.

The synchronization errors are defined as follows:

$$
\begin{aligned}
e_{i} & =x_{s i}-x_{m i}, \quad(i=1,2, \ldots, n), \\
\bar{e} & =\left[\begin{array}{llll}
x_{s 1}-x_{m 1} & x_{s 2}-x_{m 2} & \ldots & x_{s n}-x_{m n}
\end{array}\right]^{T} .
\end{aligned}
$$

Note that if the following condition is satisfied, then the objective of synchronization is realized:

$$
\|\bar{e}\| \longrightarrow 0 \text {. }
$$

Considering (1) and (2), for $i=1,2, \ldots, n$, the error dynamics can be obtained as

$$
\dot{e}_{i}=\bar{F}_{i}\left(\mathbf{X}_{m}, \mathbf{X}_{s}, t\right)+u_{i}(t)+D_{i}(t)
$$

where

$$
\begin{aligned}
\bar{F}_{i}\left(\mathbf{X}_{m}, \mathbf{X}_{s}, t\right) & =f_{s i}\left(\mathbf{X}_{s}, t\right)-f_{m i}\left(\mathbf{X}_{m}, t\right), \\
D_{i} & =\Delta f_{s i}\left(\mathbf{X}_{s}, t\right)+d_{i}(t),
\end{aligned}
$$

where $D_{i}$ denotes the lumped uncertainties.

The following Assumption is assumed to be valid throughout this study. 
Assumption 1. The lumped uncertainties $D_{i}(i=1,2, \ldots, n)$ is differentiable and satisfies $\left|D_{i}\right| \leq D_{i \max }$ and $\left|\dot{D}_{i}(t)\right| \leq D_{i \max }^{d}$, where $D_{i \text { max }}$ and $D_{i \text { max }}^{d}$ are the unknown positive constants.

2.2. Problem Description and Purposes of This Study. To satisfy condition (4), like [32], a simple sliding mode surface vector can be chosen as

$$
\mathbf{s}=\mathbf{c} \bar{e}
$$

where

$$
\begin{aligned}
& \mathbf{s}=\left[\begin{array}{llll}
s_{1} & s_{2} & \ldots & s_{n}
\end{array}\right]^{T}, \\
& \mathbf{c}=\operatorname{diag}\left(\begin{array}{llll}
c_{1} & c_{2} & \ldots & c_{n}
\end{array}\right),
\end{aligned}
$$

where $c_{i}(i=1,2, \ldots, n)$ is a positive constant.

Then, calculating the time derivative of $s_{i}(i=1,2, \ldots, n)$ along the trajectories of (5) and (7), we have

$$
\dot{s}_{i}=c_{i}\left(\bar{F}_{i}\left(\mathbf{X}_{m}, \mathbf{X}_{s}, t\right)+D_{i}+u_{i}(t)\right) \text {. }
$$

To guarantee the sliding mode surface $s_{i}$ converge to zero, it is necessary to design a robust scheme to suppress the lumped uncertainties $D_{i}$.

Then, the conventional sliding mode controller (SMC) can be designed as

$$
u_{i}(t)=\left(c_{i}\right)^{-1}\left(-\bar{F}_{i}\left(\mathbf{X}_{m}, \mathbf{X}_{s}, t\right)-k_{\mathrm{SMC} i} \operatorname{sign}\left(s_{i}\right)\right),
$$

where $\operatorname{sign}(\cdot)$ denotes the signum function, and $k_{\mathrm{SMC} i}$ is a positive constant.

Substituting (10) into (9), we have $\dot{s}_{i}=-\left(k_{\mathrm{SMC} i}-\right.$ $\left.D_{i}\right) \operatorname{sign}\left(s_{i}\right)$. Then, we can know that $s_{i} \dot{s}_{i}<0$ if $k_{\mathrm{SMC} i}>D_{i}$ and $s_{i} \neq 0$. Thus, the uncertainty $D_{i}$ can be suppressed by the discontinuous switch item $k_{\mathrm{SMC} i} \operatorname{sign}\left(s_{i}\right)$. However, the constant $k_{S M C i}$ must be selected as the upper bound of $D_{i}$, which is difficult to be obtained in advance. And the discontinuous term $k_{S M C i} \operatorname{sign}\left(s_{i}\right)$ brings undesirable chattering problem.

Recently, to avoid using the upper bound of $D_{i}$ and solve the chattering problem, some observer-based SMC schemes have been developed in [28-31].

In [26-28], the disturbance observer (DO) is designed to estimate nonvanishing disturbances and model uncertainties in the chaotic system. For the uncertainties $D_{i}(i=1,2, \ldots, n)$, the DO can be designed by using the method in [28-30]:

$$
\left\{\begin{array}{l}
\dot{Z}_{\mathrm{DO} i}=c_{i}\left(\bar{F}_{i}\left(\mathbf{X}_{m}, \mathbf{X}_{s}, t\right)+\widehat{D}_{\mathrm{DO} i}+u_{i}(t)\right) \\
\widehat{D}_{\mathrm{DO} i}=-k_{\mathrm{DO} i}\left(Z_{\mathrm{DO} i}-s_{i}\right)
\end{array}\right.
$$

where $Z_{\mathrm{DO} i}$ is the estimate state of $\mathrm{DO}, k_{\mathrm{DO} i}$ is the positive observer gain, and $\widehat{D}_{\mathrm{DO} i}$ is the estimation of $D_{i}$. Then, the DO-based SMC can be designed as

$$
u_{i}(t)=-\bar{F}_{i}\left(\mathbf{X}_{m}, \mathbf{X}_{s}, t\right)-\varepsilon_{\mathrm{DO} i} s_{i}-\sigma_{\mathrm{DO} i}\left|s_{i}\right|^{\gamma_{\mathrm{DO} i}} \operatorname{sign}\left(s_{i}\right)-\widehat{D}_{\mathrm{DO} i},
$$

where $\varepsilon_{\mathrm{DO} i}, \sigma_{\mathrm{DO} i}$, and $\gamma_{\mathrm{DO} i}$ are the positive constants, $0<\gamma_{\mathrm{DO} i}<1$. The DO (11) can guarantee the estimate error converge to the following region:

$$
\left|\widehat{D}_{\mathrm{DO} i}-D_{i}\right| \longrightarrow \frac{D_{i \max }^{d}}{\left(k_{\mathrm{DO} i} c_{i}\right)}, \quad \text { if } t \longrightarrow \infty
$$

where the constant $D_{d \max }$ is defined in Assumption 1. From (13), to achieve the high observation precision, the observer gain $k_{\mathrm{DO} i}$ should be large enough. However, from (11), it is clear that the initial value $\widehat{D}_{\mathrm{DO} i}(0)$ may be very large if $k_{\mathrm{DO} i}$ is a high gain and the initial estimate state error $Z_{\mathrm{DO} i}(0)-s_{i}(0) \neq 0$. Actually, the initial values of true state $s_{i}(0)$ cannot be known in advance for most of the cases. Thus, the initial estimate state error $Z_{\mathrm{DO} i}(0)-s_{i}(0)$ maybe not equal to 0 and even a large value. The large value $\widehat{D}_{\mathrm{DO} i}(0)$ may lead to a large overshoot control input $u_{i}(t)$ (see (12)). Then, the large overshoot $u_{i}(t)$ may reduce the dynamic performance of synchronization and even lead to the instability, and this is the undesirable spike problem of DO.

In [31], the extended state observer (ESO) is developed to estimate the uncertainties and chaotic nonlinear function. The uncertainties $D_{i}(i=1,2, \ldots, n)$ also can be estimated by using the method in [31]:

$$
\left\{\begin{array}{l}
\dot{Z}_{\mathrm{ESO} i}=c_{i}\left(\bar{F}_{i}\left(\mathbf{X}_{m}, \mathbf{X}_{s}, t\right)+\widehat{D}_{\mathrm{ESO} i}+u_{i}(t)-2 k_{\mathrm{ESO} i}\left(Z_{\mathrm{ESO} i}-s_{i}\right)\right), \\
\dot{\hat{D}}_{\mathrm{ESO} i}=-k_{\mathrm{ESO} i}^{2} c_{i}\left(Z_{\mathrm{ESO} i}-s_{i}\right),
\end{array}\right.
$$

where $Z_{\mathrm{ESO} i}$ is the estimated state of ESO, $\widehat{D}_{\mathrm{ESO} i}$ is an estimation of $D_{i}$, and $k_{\mathrm{ESO} i}$ is the observer gain of ESO. Then, the ESO-based SMC can be designed as

$$
\begin{aligned}
u_{i}(t)= & -\bar{F}_{i}\left(X_{m}, X_{s}, t\right)-\varepsilon_{\mathrm{ESO} i} s_{i}-\sigma_{\mathrm{ESO} i}\left|s_{i}\right|^{\gamma_{\mathrm{ESO} i} \operatorname{sign}\left(s_{i}\right)} \\
& -\widehat{D}_{\mathrm{ESO} i},
\end{aligned}
$$

where $\varepsilon_{\mathrm{ESO} i}, \sigma_{\mathrm{ESO} i}$, and $\gamma_{\mathrm{ESO} i}$ are the positive constants, $0<\gamma_{\mathrm{ESO} i}<1$. The ESO can guarantee the estimate error converge to following region:

$$
\left|\widehat{D}_{\mathrm{ESO} i}-D_{i}\right| \longrightarrow \frac{D_{i \max }^{d}}{\left(k_{\mathrm{ESO} i} c_{i}\right)}, \quad \text { if } t \longrightarrow \infty \text {. }
$$

Define the estimate error vector $\mathbf{h}=\left[\begin{array}{lll}Z_{\mathrm{ESO} i}-s_{i} & \widehat{D}_{\mathrm{ESO} i}\end{array}\right.$ $\left.-D_{i}\right]^{T}$. From ESO (14), we have

$$
\dot{\mathbf{h}}=\mathbf{A}_{h} \mathbf{h}+\Delta,
$$

where

$$
\begin{aligned}
\mathbf{A}_{h} & =c_{i}\left[\begin{array}{cc}
-2 k_{\mathrm{ESO} i} & 1 \\
-k_{\mathrm{ESO} i}^{2} & 0
\end{array}\right], \\
\boldsymbol{\Delta} & =\left[\begin{array}{c}
0 \\
\dot{D}_{i}
\end{array}\right] .
\end{aligned}
$$

The solution to the differential equation (17) can be easily obtained as 


$$
\mathbf{h}=\mathbf{e}^{\mathbf{A}_{h} t} \mathbf{h}(0)+\int_{0}^{t} \mathbf{e}^{\mathbf{A}_{h}(t-\tau)} \dot{D}_{i}(\tau) \mathrm{d} \tau,
$$

where $e$ is the $e$ constant. Let $\left[\begin{array}{lll}* 20 c \bar{h}_{1} & \bar{h}_{2}\end{array}\right]^{T}=\int_{0}^{t} \mathbf{e}^{\mathbf{A}_{h}(t-\tau)}$ $\dot{D}_{i}(\tau) \mathrm{d} \tau$. Expanding $e^{\mathbf{A}_{h} t} \mathbf{h}(0)$, the estimate error can be rewritten as

$$
\begin{aligned}
\widehat{D}_{\mathrm{ESO} i}-D_{i}= & -\left(k_{\mathrm{ESO} i} c_{i}\right)^{2} t e^{-k_{\mathrm{ESO} i} c_{i} t}\left(Z_{\mathrm{ESO} i}(0)-s_{i}(0)\right) \\
& +\left(k_{\mathrm{ESO} i} c_{i} t+1\right) e^{-k_{\mathrm{ESO} i} c_{i} t}\left(\widehat{D}_{\mathrm{ESO} i}(0)-D_{i}(0)\right)+\bar{h}_{2}
\end{aligned}
$$

For the small time $t=1 /\left(k_{\mathrm{ESO} i} c_{i}\right)$, we have

$$
\begin{aligned}
\widehat{D}_{\mathrm{ESO} i}= & -k_{\mathrm{ESO} i} c_{i}\left(Z_{\mathrm{ESO} i}(0)-s_{i}(0)\right) e^{-1} \\
& +2 e^{-1}\left(\widehat{D}_{\mathrm{ESO} i}(0)-D_{i}(0)\right)+D_{i} \\
& +\bar{h}_{2}, \quad \text { if } t=\frac{1}{\left(k_{\mathrm{ESO} i} c_{i}\right)} .
\end{aligned}
$$

It can be known that $\left(k_{\mathrm{ESO} i} c_{i}\right)\left(Z_{\mathrm{ESO} i}(0)-s_{i}(0)\right)$ is a very large value if $k_{\mathrm{ESO} i}$ is a high gain and $Z_{\mathrm{ESO} i}(0)-s_{i}(0) \neq 0$. Thus, the undesirable spike problem also exists in ESO.

2.2.1. Motivation of This Study. From the previous discussion, an undesirable spike problem can be caused by the high observer gain in ESO and DO if the initial values of estimate and true states are not equal. Thus, if the initial value of true states is unknown, to avoid the spike problem, the ESObased and DO-based controller cannot adopt the high observer gains to guarantee the control precision. Actually, the initial value of true states cannot be known in advance in most of cases. This motivates the research topic of this study, that is, for the chaotic system in the presence of uncertainties, designing a new TVGDO and TVGDO-based SMC schemes not only can guarantee high control precision but also eliminate the undesirable spike problem.

\section{Main Result}

3.1. Observer Design and Stability Analysis. In this section, a novel time-varying gain disturbance observer (TVGDO) will be proposed. The TVGDO can guarantee the high precision and avoid the undesirable spike problem even if the initial values of estimate and true states are not equal.

The expression of TVGDO and the stability analysis are given in the following Theorem.

Theorem 1. Taking the master and slave chaotic systems (1) and (2) into consideration, for the uncertainties $D_{i}(i=1,2, \ldots, n)$, the TVGDO (22) is constructed.

$$
\left\{\begin{array}{l}
\dot{Z}_{i}=c_{i}\left(\bar{F}_{i}\left(\mathbf{X}_{m}, \mathbf{X}_{s}, t\right)+\widehat{D}_{i}+u_{i}(t)\right) \\
\widehat{D}_{i}=-k_{i}(t)\left(Z_{i}-s_{i}\right)+\int_{0}^{t} \dot{k}_{i}(\tau)\left(Z_{i}-s_{i}\right) \mathrm{d} \tau \\
k_{i}(t)=\eta_{i 1}\left(1-e^{-\eta_{i 2} t}\right), \\
\dot{k}_{i}(\tau)=\eta_{i 1} \eta_{i 2} e^{-\eta_{i 2} \tau}
\end{array}\right.
$$

where $\eta_{i 1}$ and $\eta_{i 2}$ are the positive constants, and $k_{i}(t)$ is a nonnegative time-varying gain. Assumption 1 is valid. The estimate error of TVGDO is defined as $\bar{D}_{i}=\widehat{D}_{i}-D_{i}$. Then, the estimate error $\bar{D}_{i}$ will converge to the following region:

$$
\left|\bar{D}_{i}\right| \leq \frac{D_{i \max }^{d}}{\left(\eta_{i 1} c_{i}\right)}, \quad \text { if } t \longrightarrow \infty \text {. }
$$

Proof. The estimate error of TVGDO is defined as $\bar{D}_{i}=\widehat{D}_{i}-D_{i}(i=1,2, \ldots, n)$. Differentiating $\bar{D}_{i}$ gives

$$
\overline{\bar{D}}_{i}=\dot{\hat{D}}_{i}-\dot{D}_{i}
$$

Considering (9) and (22), (24) can be rewritten as

$$
\begin{aligned}
\dot{\overline{D_{i}}} & =-\dot{k}_{i}(t)\left(Z_{i}-s_{i}\right)-k_{i}(t)\left(\dot{Z}_{i}-\dot{s}_{i}\right)+\dot{k}_{i}(t)\left(Z_{i}-s_{i}\right)-\dot{D}_{i} \\
& =-k_{i}(t)\left(\dot{Z}_{i}-\dot{s}_{i}\right)-\dot{D}_{i} \\
& =-k_{i}(t)\left(c_{i}\left(\bar{F}_{i}\left(\mathbf{X}_{m}, \mathbf{X}_{s}, t\right)+\widehat{D}_{i}+u_{i}(t)\right)-c_{i}\left(\bar{F}_{i}\left(\mathbf{X}_{m}, \mathbf{X}_{s}, t\right)+D_{i}+u_{i}(t)\right)\right)-\dot{D}_{i} \\
& =-k_{i}(t) c_{i}\left(\widehat{D}_{i}-D_{i}\right)-\dot{D}_{i} \\
& =-k_{i}(t) c_{i} \bar{D}_{i}-\dot{D}_{i} .
\end{aligned}
$$

Construct the Lyapunov function $J_{i}$ as

$$
J_{i}=\bar{D}_{i}^{2} / 2
$$

Then, calculating the time derivative of $J_{i}$ along the trajectory of (25), we get

$$
\dot{J}_{i}=\bar{D}_{i} \dot{D_{i}}=-k_{i}(t) c_{i}\left|\bar{D}_{i}\right|^{2}-\dot{D}_{i} \bar{D}_{i} .
$$

Considering Assumption 1, we have

$$
\begin{aligned}
\dot{J}_{i} \leq & -k_{i}(t) c_{i}\left|\bar{D}_{i}\right|^{2}+D_{i \max }^{d}\left|\bar{D}_{i}\right|=-2 k_{i}(t) c_{i} J_{i} \\
& +\sqrt{2} D_{i \max }^{d} J_{i}^{1 / 2}, \quad \text { if } t \geq 0 .
\end{aligned}
$$

Since $k_{i}(t) \geq 0$, from (28), it can be known that

$$
\dot{J}_{i} \leq \sqrt{2} D_{i \max }^{d} J_{i}^{1 / 2}, \quad \text { if } t \geq 0 .
$$

Then, we have 


$$
J_{i}^{-1 / 2} \dot{J}_{i} \leq \sqrt{2} D_{i \max }^{d}, \quad \text { if } t \geq 0 .
$$

Integrating (30) gives

$$
2 J_{i}^{1 / 2} \leq \sqrt{2} D_{i \max }^{d} t+2 J_{\mathrm{i}}^{1 / 2}(0), \quad \text { if } t \geq 0 .
$$

From the expression of $k_{i}(t)$, it can be known that the following condition can be satisfied in an arbitrary finite time $t_{i f}$ :

$$
k_{i}(t) \geq k_{i}\left(t_{i f}\right)>0, \quad \text { if } t \geq t_{i f} .
$$

Combining (28) and (32), we have

$$
\dot{J}_{i} \leq-2 k_{i}(t) c_{i} J_{i}+\sqrt{2} D_{i \max }^{d} J_{i}^{1 / 2}, \quad \text { if } t \geq t_{i f} .
$$

From (31), for an arbitrary finite time $t_{i f}$, it is clear that $J_{i}^{1 / 2}\left(t_{i f}\right)$ is bounded. If $J_{i}^{1 / 2}>D_{i \max }^{d} /\left(\sqrt{2} k_{i}(t) c_{i}\right)$, it also can be known from (33) that $\dot{j}_{i}<0$. Then, we have

$$
J_{i}^{1 / 2} \leq \frac{D_{i \max }^{d}}{\left(\sqrt{2} k_{i}(t) c_{i}\right)}, \quad \text { if } t \longrightarrow \infty .
$$

Consider $\lim _{t \rightarrow \infty} k_{i}(t)=\eta_{i 1}$; then, we have

$$
J_{i}^{1 / 2} \leq \frac{D_{i \max }^{d}}{\left(\sqrt{2} \eta_{i 1} c_{i}\right)}, \quad \text { if } t \longrightarrow \infty .
$$

From (35) and $\sqrt{2} J_{i}^{1 / 2}=\left|\bar{D}_{i}\right|$, we have

$$
\left|\bar{D}_{i}\right| \leq \frac{D_{i \max }^{d}}{\left(\eta_{i 1} c_{i}\right)}, \quad \text { if } t \longrightarrow \infty .
$$

The proof is finished.

3.2. The Spike-Free Characteristic Analysis. Let $\int_{0}^{t} k_{i}(\tau)$ $c_{i} \mathrm{~d} \tau=k_{i S}(t)$. Considering (22) and solving the differential equation (25), the solution of estimate error $\bar{D}_{i}$ in time domain can be easily obtained as

$$
\bar{D}_{i}=e^{-k_{i S}(t)}\left(\widehat{D}_{i}(0)-D_{i}(0)-\int_{0}^{t} \dot{D}_{i}(\tau) e^{k_{i S}(\tau)} \mathrm{d} \tau\right),
$$

where $\mathrm{e}$ is the $e$ constant. Substituting the detailed expression of $\widehat{D}_{i}(0)$ into (37), we have

$$
\begin{aligned}
\bar{D}_{i} & =e^{-k_{i S}(t)}\left(\widehat{D}_{i}(0)-D_{i}(0)\right)-e^{-k_{i S}(t)} \int_{0}^{t} \dot{D}_{i}(\tau) e^{k_{i S}(\tau)} \mathrm{d} \tau \\
& =e^{-k_{i S}(t)}\left(-k_{i}(0)\left(Z_{i}(0)-s_{i}(0)\right)+\int_{0}^{0} \dot{k}_{i}(\tau)\left(Z_{i}-s_{i}\right) \mathrm{d} \tau-D_{i}(0)\right)-e^{-k_{i S}(t)} \int_{0}^{t} \dot{D}_{i}(\tau) e^{k_{i S}(\tau)} \mathrm{d} \tau .
\end{aligned}
$$

From the previous discussion in Section 2.2, it can be known that the undesirable spike problem is caused by the spike term $k_{\mathrm{DO} i}\left(Z_{\mathrm{DO} i}(0)-s_{i}(0)\right)$ in DO (11) or the spike term $\left(k_{\mathrm{ESO} i} c_{i}\right)\left(Z_{\mathrm{ESO} i}(0)-s_{i}(0)\right)$ in ESO (14). Since $k_{i}(0)\left(Z_{i}(0)-s_{i}(0)\right)=0$ and $\int_{0}^{0} \dot{k}_{i}(\tau)\left(Z_{i}-s_{i}\right) \mathrm{d} \tau=0$, it is clear that the expression of $\bar{D}_{i}$ in (38) does not contain any spike term. Thus, the spike problem is avoided in the TVGDO.

Remark 1. It can be known from (26) that a small enough estimate error can be guaranteed by choosing $\eta_{i 1}$ reasonably. Thus, the proposed TVGDO not only can eliminate the undesirable spike problem but also can guarantee high observation precision.

3.3. Observer-Based Controller Design and Stability Analysis. Then, a novel TVGDO-based sliding mode controller will be developed in this section. The expression of the proposed controller and the stability analysis are given in the following Theorem.

Theorem 2. Taking the master and slave chaotic systems (1) and (2) into consideration, for $i=1,2, \ldots, n$, the TVGDObased sliding mode controller is constructed as

$$
u_{i}(t)=-\bar{F}_{i}\left(\mathbf{X}_{m}, \mathbf{X}_{s}, t\right)-\varepsilon_{i} s_{i}-\sigma_{i}\left|s_{i}\right|^{\gamma_{i}} \operatorname{sign}\left(s_{i}\right)-\widehat{D}_{i},
$$

where the sliding mode surface $s_{i}$ is defined in (7). $\varepsilon_{i}, \sigma_{i}$, and $\gamma_{i}$ are the positive constants. $0<\gamma_{i}<1 . \widehat{D}_{i}$ is given in
TVGDO (22). Assumption 1 is valid. The synchronization error $e_{i}$ can converge to following small region:

$$
\left|e_{i}\right| \leq \min \left\{\frac{\left(D_{i \max }^{d} /\left(\varepsilon_{i} \eta_{i 1} c_{i}\right)\right)}{c_{i}}, \frac{\left(D_{i \max }^{d} /\left(\sigma_{i} \eta_{i 1} c_{i}\right)\right)^{1 / \gamma_{i}}}{c_{i}}\right\}, \quad \text { if } t \longrightarrow \infty .
$$

Proof. Construct the Lyapunov function $P_{i}(i=1,2, \ldots, n)$ as

$$
P_{i}=\frac{s_{i}^{2}}{2}
$$

Then, calculating the time derivative of $P_{i}$ along the trajectory of (9), we get

$$
P_{i}=s_{i} \dot{s}_{i}=s_{i}\left(c_{i}\left(\bar{F}_{i}\left(\mathbf{X}_{m}, \mathbf{X}_{s}, t\right)+D_{i}+u_{i}(t)\right)\right) .
$$

Substituting the control input (39) into (42), we have

$$
\begin{aligned}
\dot{P}_{i} & =c_{i} s_{i}\left(D_{i}-\varepsilon_{i} s_{i}-\sigma_{i}\left|s_{i}\right|^{\gamma_{i}} \operatorname{sign}\left(s_{i}\right)-\widehat{D}_{i}\right) \\
& =c_{i}\left(-\varepsilon_{i} s_{i}^{2}-\sigma_{i}\left|s_{i}\right|^{\gamma_{i}+1}-\left(\widehat{D}_{i}-D_{i}\right) s_{i}\right) \\
& =-\varepsilon_{i} c_{i} s_{i}^{2}-\sigma_{i} c_{i}\left|s_{i}\right|^{\gamma_{i}+1}-c_{i} \bar{D}_{i} s_{i} \\
& \leq-2 \varepsilon_{i} c_{i} P_{i}-2^{\left(\gamma_{i}+1 / 2\right)} \sigma_{i} c_{i} P_{i}^{\left(\gamma_{i}+1 / 2\right)}+c_{i} \sqrt{2}\left|\bar{D}_{i}\right| P_{i}^{1 / 2},
\end{aligned}
$$

where the estimate error $\bar{D}_{i}=\widehat{D}_{i}-D_{i}$. 
From (43), we know that $e_{i}$ affected the estimate error $\bar{D}_{i}$. Thus, the following proof will consist of two steps. In the first step, it will be proved that $e_{i}$ will not escape to infinity in arbitrary finite time (before $\bar{D}_{i}$ converges to a neighborhood of zero). In the second step, it will be proved that $e_{i}$ will converge to a neighborhood of zero after $\bar{D}_{i}$ converges to a small neighborhood of zero.

Step 1. From (31) in Theorem 1, we have known that the estimate error $\bar{D}_{i}$ is bounded as

$$
2 J_{i}^{1 / 2} \leq \sqrt{2} D_{i \max }^{d} t+2 J_{i}^{1 / 2}(0), \quad \text { if } t \geq 0 .
$$

For the arbitrary finite time $t \leq t_{s}$, it can be known that $\left|\bar{D}_{i}\right| \leq D_{i \max }^{d} t+\sqrt{2} J_{i}^{1 / 2}(0) \leq D_{i \max }^{d}\left(t_{s}\right)+\sqrt{2} J_{i}^{1 / 2}(0), \quad$ if $t \leq t_{s}$.

Let the positive constant $D_{P i}=D_{i \max }^{d}\left(t_{s}\right)+\sqrt{2} J_{i}^{1 / 2}(0)$. Then, combining (43) with (45), we have

$$
\begin{aligned}
\dot{P}_{i} & \leq-2 \varepsilon_{i} c_{i} P_{i}+c_{i} \sqrt{2} D_{P i} P_{i}^{1 / 2} \\
& \leq P_{i}^{1 / 2}\left(-2 \varepsilon_{i} c_{i} P_{i}^{1 / 2}+c_{i} \sqrt{2} D_{P i}\right), \quad \text { if } t \leq t_{s}, \\
\dot{P}_{i} & \leq-2^{\left(\gamma_{i}+1 / 2\right)} \sigma_{i} c_{i} P_{i}^{\left(\gamma_{i}+1 / 2\right)}+c_{i} \sqrt{2} D_{P i} P_{i}^{1 / 2} \\
& =P_{i}^{1 / 2}\left(-2^{\left(\gamma_{i}+1 / 2\right)} \sigma_{i} c_{i} P_{i}^{\left(\gamma_{i} / 2\right)}+c_{i} \sqrt{2} D_{P i}\right), \quad \text { if } t \leq t_{s} .
\end{aligned}
$$

From (46) and (47), we can know that $\dot{P}_{i}<0$ if $P_{i}>\left(D_{P_{i}} /\left(2^{\left(\gamma_{i} / 2\right)} \sigma_{i}\right)\right)^{\left(\gamma_{i} / 2\right)}$ or $P_{i}>\left(D_{P i} /\left(\sqrt{2} \varepsilon_{i}\right)\right)^{2}$. Thus, for $t \leq t_{s}, P_{i}$ will not escape to infinity and is bounded as

$$
\left|P_{i}\right| \leq \max \left\{\left(\frac{D_{P i}}{\left(2^{\left(\gamma_{i} / 2\right)} \sigma_{i}\right)}\right)^{\left(2 / \gamma_{i}\right)},\left(\frac{D_{P i}}{\left(\sqrt{2} \varepsilon_{i}\right)}\right)^{2}\right\}, \quad \text { if } t \leq t_{s} .
$$

Let the constant $P_{i \max }=\max \left\{\left(D_{P i} /\left(2^{\left(\gamma_{i} / 2\right)} \sigma_{i}\right)\right)^{\left(2 / \gamma_{i}\right)}\right.$, $\left.\left(D_{P i} /\left(\sqrt{2} \varepsilon_{i}\right)\right)^{2}\right\}$. Then, we can know that $e_{i}$ will not escape to infinity and is bounded as $\left|e_{i}\right| \leq \sqrt{2 P_{i \max }} / c_{i}$ if $t \leq t_{s}$.

Step 2. From (43), we have

$$
\begin{aligned}
& \dot{P}_{i} \leq-\sqrt{2} c_{i} P_{i}^{1 / 2}\left(\sqrt{2} \varepsilon_{i} P_{i}^{1 / 2}-\left|\bar{D}_{i}\right|\right), \\
& \dot{P}_{i} \leq-\sqrt{2} c_{i} P_{i}^{1 / 2}\left(2^{\left(\gamma_{i} / 2\right)} \sigma_{i} P_{i}^{\left(\gamma_{i} / 2\right)}-\left|\bar{D}_{i}\right|\right) .
\end{aligned}
$$

Then, we can know that

$$
\begin{aligned}
& \dot{P}_{i}<0, \quad \text { if } \sqrt{2} \varepsilon_{i} P_{i}^{1 / 2}>\left|\bar{D}_{i}\right| \geq 0, \\
& \dot{P}_{i}<0, \quad \text { if } 2^{\left(\gamma_{i} / 2\right)} \sigma_{i} P_{i}^{\left(\gamma_{i} / 2\right)}>\left|\bar{D}_{i}\right| \geq 0 .
\end{aligned}
$$

According to (50) and (51), we have

$$
\begin{array}{ll}
P_{i} \leq\left(\frac{\left|\bar{D}_{i}\right|}{\left(\sqrt{2} \varepsilon_{i}\right)}\right)^{2}, \quad \text { if } t \longrightarrow \infty, \\
P_{i} \leq\left(\frac{\left|\bar{D}_{i}\right|}{\left(2^{\left(\gamma_{i} / 2\right)} \sigma_{i}\right)}\right)^{\left(2 / \gamma_{i}\right)}, \quad \text { if } t \longrightarrow \infty .
\end{array}
$$

From Theorem 1, it can be known that the estimate error $\bar{D}_{i}$ is bounded as

$$
\left|\bar{D}_{i}\right| \leq \frac{D_{i \max }^{d}}{\left(\eta_{i 1} c_{i}\right)}, \quad \text { if } t \longrightarrow \infty \text {. }
$$

Combining (52)-(54), we have

$$
P_{i} \leq \min \left\{\left(\frac{D_{i \max }^{d}}{\left(\sqrt{2} \varepsilon_{i} \eta_{i 1} c_{i}\right)}\right)^{2},\left(\frac{D_{i \max }^{d}}{\left(2^{\left(\gamma_{i} / 2\right)} \sigma_{i} \eta_{i 1} c_{i}\right)}\right)^{\left(2 / \gamma_{i}\right)}\right\}, \quad \text { if } t \longrightarrow \infty \text {. }
$$

From (55), it can be known that $s_{i}$ will converge to following region:

$$
\left|s_{i}\right| \leq \min \left\{\left(\frac{D_{i \max }^{d}}{\left(\varepsilon_{i} \eta_{i 1} c_{i}\right)}\right),\left(\frac{D_{i \max }^{d}}{\left(\sigma_{i} \eta_{i 1} c_{i}\right)}\right)^{\left(1 / \gamma_{i}\right)}\right\}, \quad \text { if } t \longrightarrow \infty .
$$

Then, the synchronization error will converge to following small region:

$$
\left|e_{i}\right| \leq \min \left\{\frac{\left(D_{i \max }^{d} /\left(\varepsilon_{i} \eta_{i 1} c_{i}\right)\right)}{c_{i}}, \frac{\left(D_{i \max }^{d} /\left(\sigma_{i} \eta_{i 1} c_{i}\right)\right)^{\left(1 / \gamma_{i}\right)}}{c_{i}}\right\}, \quad \text { if } t \longrightarrow \infty .
$$

The proof is finished.

Remark 2. It can be known from (57) that, if large enough observer parameter $\eta_{i 1}$ and the control parameters $\sigma_{i}$, $\varepsilon_{i}$, and $c_{i}$ are chosen, then the convergence region of synchronization error will be small enough. It means that the synchronization error can be made arbitrarily small through adjusting parameters properly.

\section{Simulation Results}

In this section, to illustrate the effectiveness of the proposed methods, the mathematical simulation is presented. The master and slave chaotic systems are selected as the threedimensional chaotic systems given in [32]. Thus, for systems (1) and (2), we select $n=3$. The chaotic nonlinear function and uncertainties are chosen as 


$$
\left\{\begin{array}{l}
f_{m 1}\left(X_{m}, t\right)=\frac{40}{14} x_{m 1}-x_{m 2} x_{m 3}+10 \\
f_{m 2}\left(X_{m}, t\right)=-10 x_{m 2}+x_{m 1} x_{m 3} \\
f_{m 3}\left(X_{m}, t\right)=-4 x_{m 3}+x_{m 1} x_{m 2}, \\
f_{s 1}\left(X_{s}, t\right)=\frac{40}{14} x_{s 1}-x_{s 2} x_{s 3}+10 \\
f_{s 2}\left(X_{s}, t\right)=-10 x_{s 2}+x_{s 1} x_{s 3} \\
f_{s 3}\left(X_{s}, t\right)=-4 x_{s 3}+x_{s 1} x_{s 2}, \\
\Delta f_{s 1}\left(X_{s}, t\right)=3 \% x_{s 1}+5 \% x_{s 2}, \\
\Delta f_{s 2}\left(X_{s}, t\right)=2 \% x_{s 1}+7 \% x_{s 2}+3 \% x_{s 3} \\
d_{2}(t)=50 \cos \left(\frac{t}{5}\right)+20 \\
d_{s 3}\left(X_{s}, t\right)=-2 \% x_{s 1}+3 \% x_{s 2}+6 \% x_{s 3} \\
d_{1}(t)=60 \cos \left(\frac{t}{5}\right)+20
\end{array}\right.
$$

The initial system states are set as $x_{m 1}(0)=4$, $x_{m 2}(0)=3.5, x_{m 3}(0)=2.5$, and $x_{s 1}(0)=x_{s 2}(0)=x_{s 3}(0)$ $=0$. The simulation method is chosen as the fixed step Dormand-Prince method. The step size of simulation is set as $0.001 \mathrm{~s}$. Let $u_{i}(t)=0(i=1,2,3)$, and the chaotic behavior of the master system (1) is shown in Figure 1.

For the comparison, the proposed TVGDO-based SMC (46), DO-based SMC (12), and ESO-based SMC (15) are considered in this section.

Case 1. (Initial values of estimate and true states are equal). In this case, the SMC parameters of the three methods are selected as $c_{i}=1, \varepsilon_{\mathrm{DO} i}=2, \varepsilon_{\mathrm{ESO} i}=0.5, \varepsilon_{i}=0.5, \sigma_{\mathrm{DO} i}=12$, $\sigma_{\mathrm{ESO} i}=7, \quad \sigma_{i}=2$, and $\gamma_{\mathrm{DO} i}=\gamma_{\mathrm{ESO} i}=\gamma_{i}=0.7(i=1,2,3)$. Then, it can be known that the initial values of sliding mode surface are $s_{1}(0)=-4, s_{2}(0)=-3.5$, and $s_{3}(0)=-2.5$. The observer gains of DO and ESO are chosen as $k_{\mathrm{DO} i}=50$ and $k_{\mathrm{ESO} i}=50(i=1,2,3)$, respectively. The time-varying parameters are chosen as $\eta_{i 1}=50$ and $\eta_{i 1}=25(i=1,2,3)$. Thus, the observer gain of TVGDO $k_{i}(t)(i=1,2,3)$ is close to 50 . And, in this case, we consider that the initial value of sliding mode surface $s_{i}(0)(i=1,2,3)$ is known. Then, the initial values of estimate states in DO, ESO, and TVGDO can be chosen as

$$
\left\{\begin{array}{l}
Z_{\mathrm{DO} 1}=s_{1}(0)=-4, \\
Z_{\mathrm{DO} 2}=s_{2}(0)=-3.5, \\
Z_{\mathrm{DO} 3}=s_{3}(0)=-2.5, \\
Z_{\mathrm{ESO} 1}=s_{1}(0)=-4, \\
Z_{\mathrm{ESO} 2}=s_{2}(0)=-3.5, \\
Z_{\mathrm{ESO} 3}=s_{3}(0)=-2.5, \\
Z_{1}=s_{1}(0)=-4, \\
Z_{2}=s_{2}(0)=-3.5, \\
Z_{3}=s_{3}(0)=-2.5 .
\end{array}\right.
$$

Thus, for DO, ESO, and TVGDO, the initial values of estimate and true states are equal. Figures 2-5 show the simulation results for Case1. From Figure 2, it is clear that the DO-based SMC, ESO-based SMC, and proposed TVGDO-based SMC all can guarantee the synchronization errors that converge to a small neighborhood of zero. Figure 3 shows that DO, ESO, and TVGDO can ensure the estimation errors converge to a small neighborhood of zero. From Figure 4, it is clear that the control inputs of the three observer-based sling mode controllers are chattering-free. Thus, the undesirable chattering problem in conventional SMC can be solved by employing these observer-based schemes. Figure 5 shows the time-varying observer gains of proposed TVGDO. Thus, the results in Case 1 proved that the control parameters used in Case 1 for the three methods can achieve a similar good performance when the initial values of estimate and true states are equal.

Case 2. (The initial values of estimate and true states are not equal). From the simulation result of Case 1, we have known that the three methods can achieve a similar control performance under the control parameters chosen in Case 1. To ensure the fairness of comparison, the control parameters are selected, the same parameters as in Case 1. And, in this case, we consider that the initial value $s_{i}(0)(i=1,2,3)$ is unknown. The initial values of estimate states of DO, ESO, and TVGDO are chosen as

$$
\left\{\begin{array}{l}
Z_{\mathrm{DO} 1}=0 \neq s_{1}(0), \\
Z_{\mathrm{DO} 2}=0 \neq s_{2}(0), \\
Z_{\mathrm{DO} 3}=0 \neq s_{3}(0), \\
Z_{\mathrm{ESO} 1}=0 \neq s_{1}(0), \\
Z_{\mathrm{ESO} 2}=0 \neq s_{2}(0), \\
Z_{\mathrm{ESO} 3}=0 \neq s_{3}(0) \\
Z_{1}=0 \neq s_{1}(0), \\
Z_{2}=0 \neq s_{2}(0), \\
Z_{3}=0 \neq s_{3}(0),
\end{array}\right.
$$

Thus, for DO, ESO, and TVGDO, the initial values of estimate and true states are not equal. 

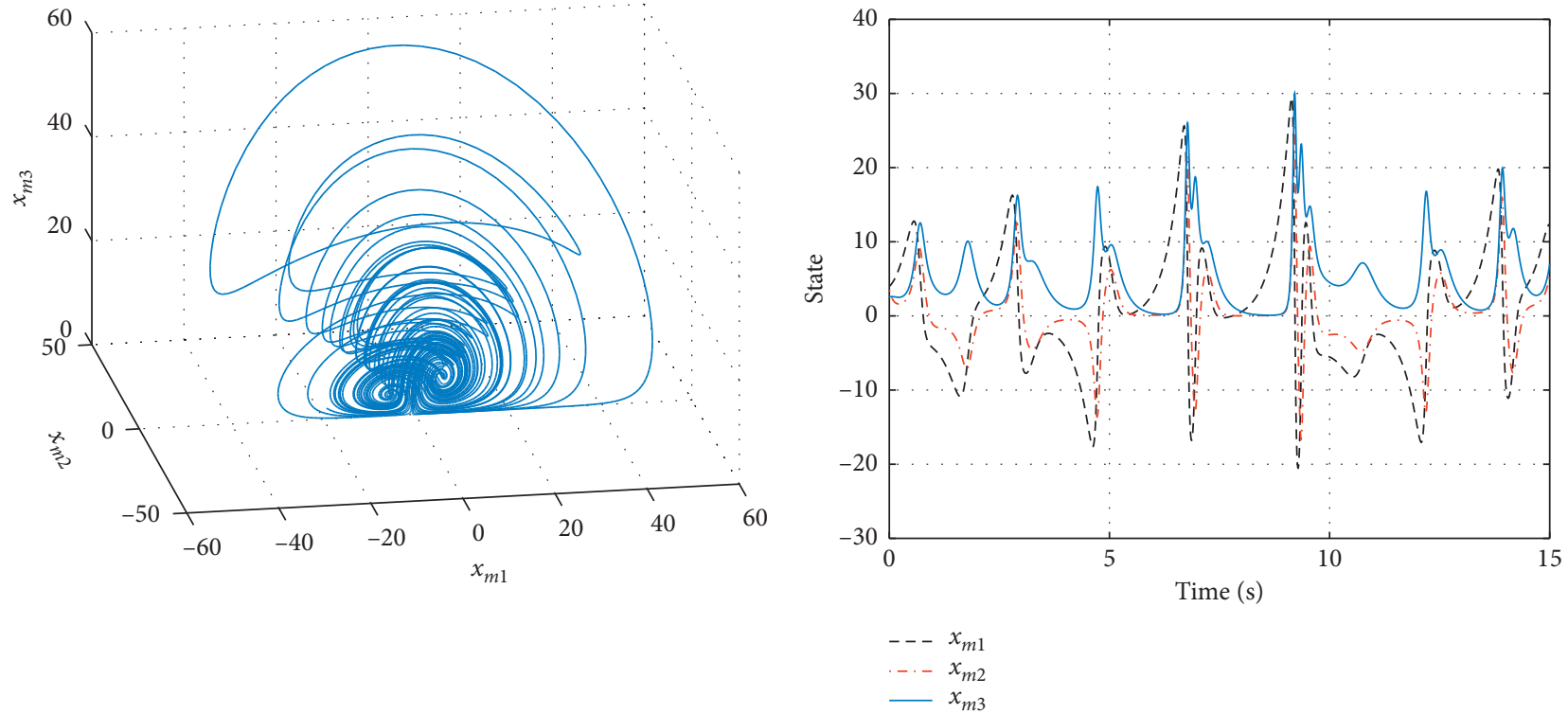

(a)

(b)

FIGURE 1: Chaotic behavior of the system without the control input. (a) Phase portrait of the system. (b) System states $x_{1}$ and $x_{2}$.
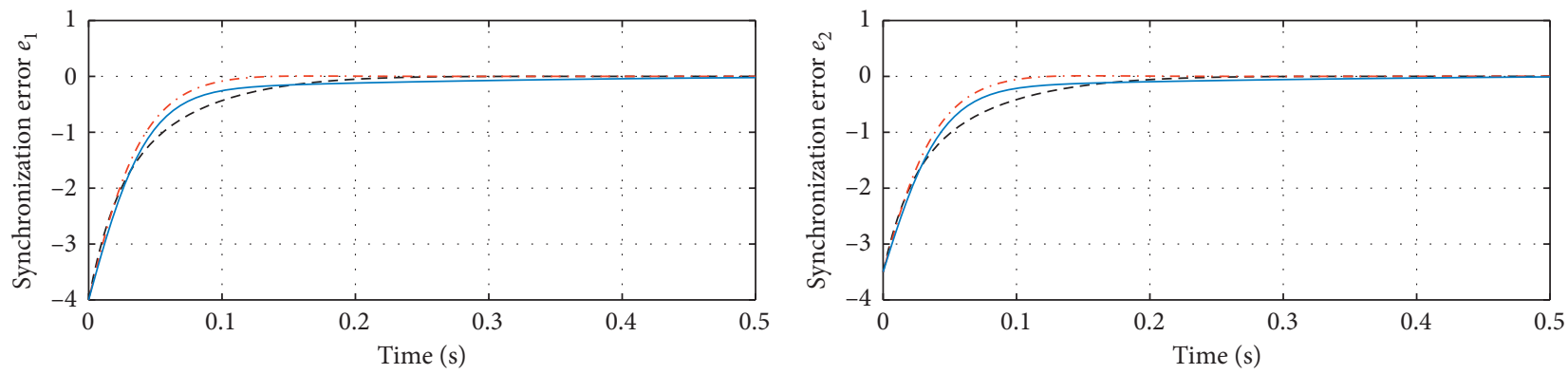

- - - DO-based SMC

- . - DO-based SMC

-.- ESO-based SMC

-.- ESO-based SMC

— Proposed TVGDO-based SMC

(a)

(b)

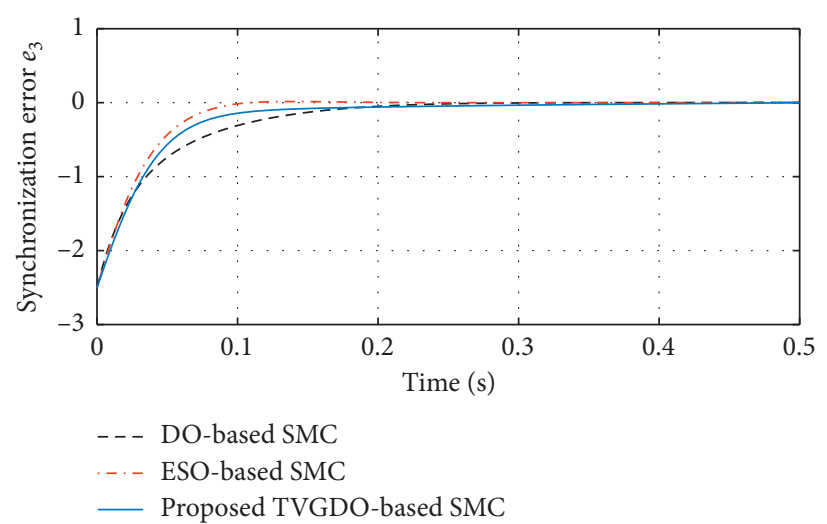

(c)

FIgURE 2: Synchronization error (Case 1). 

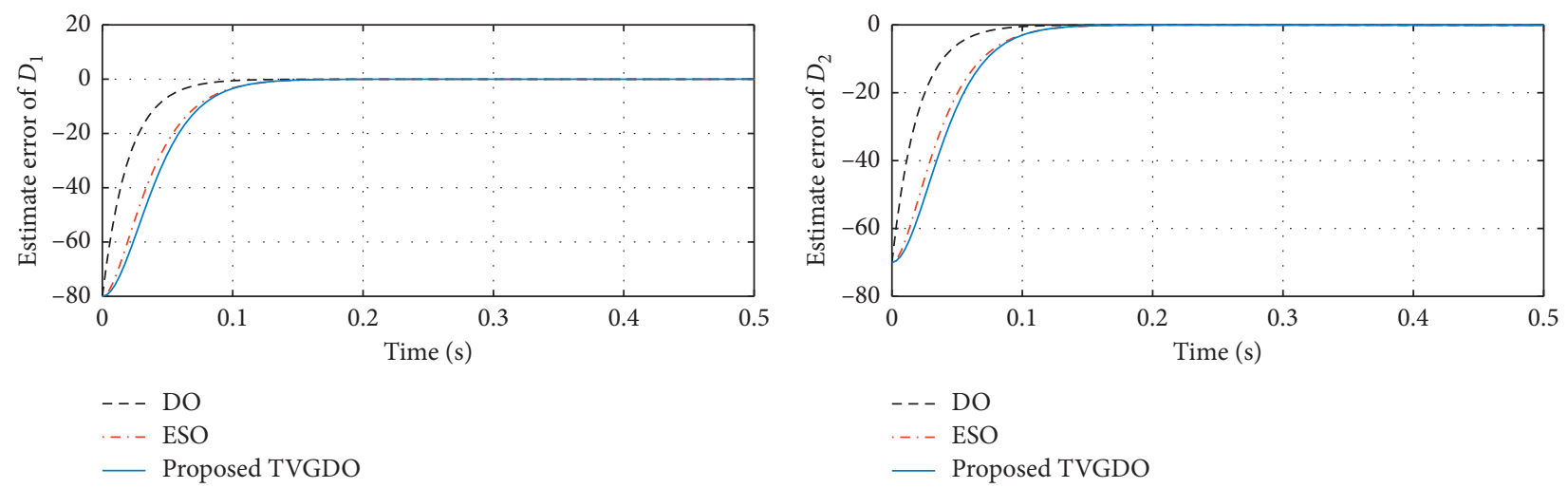

- - DO

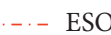

— Proposed TVGDO

(a)

(b)

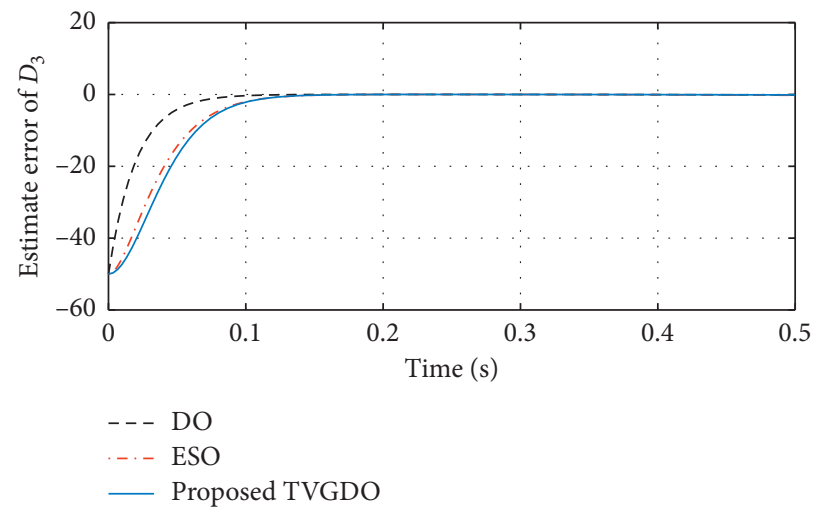

(c)

Figure 3: Estimate error (Case 1).

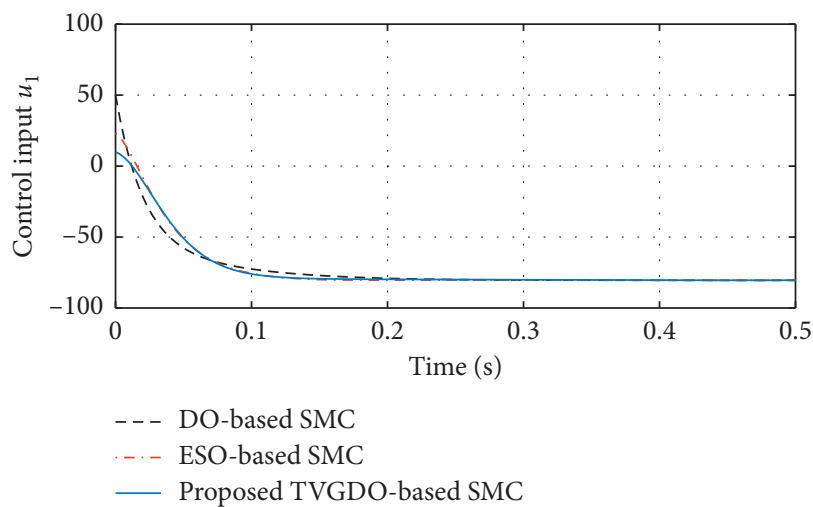

(a)

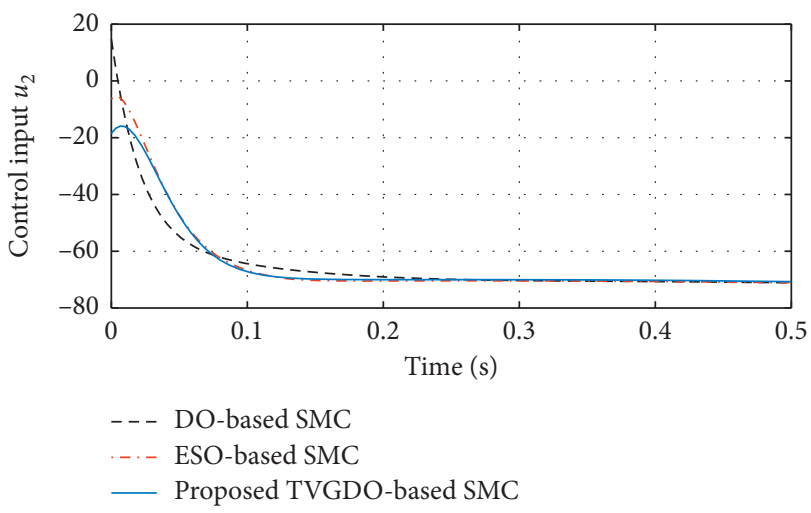

(b)

Figure 4: Continued. 


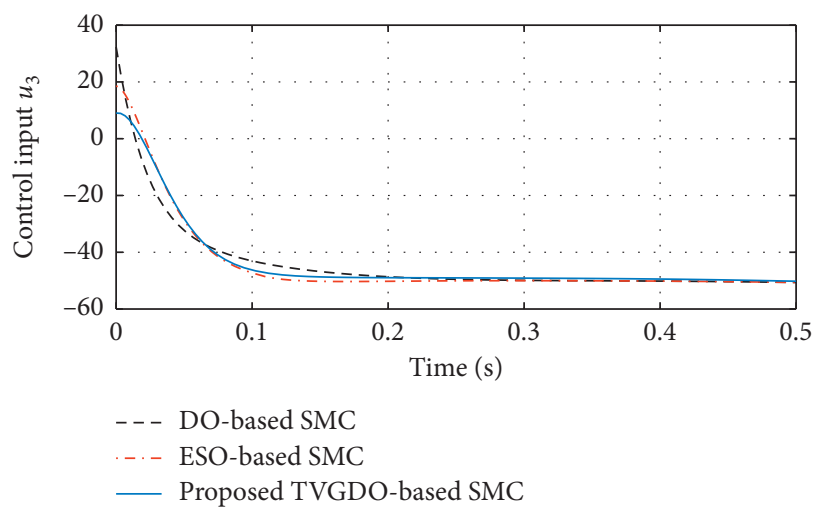

(c)

Figure 4: Control input (Case 1).

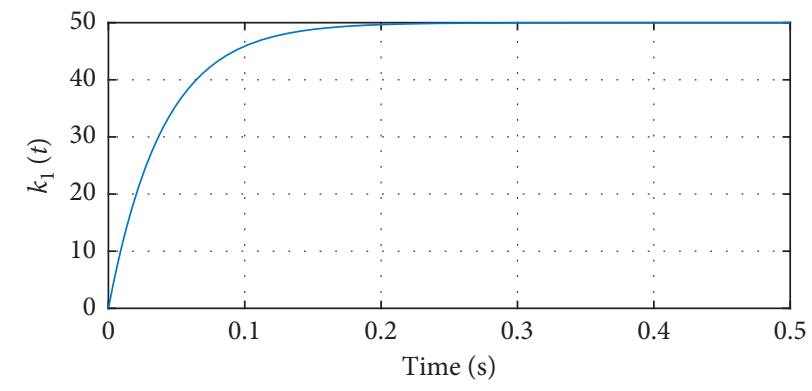

(a)

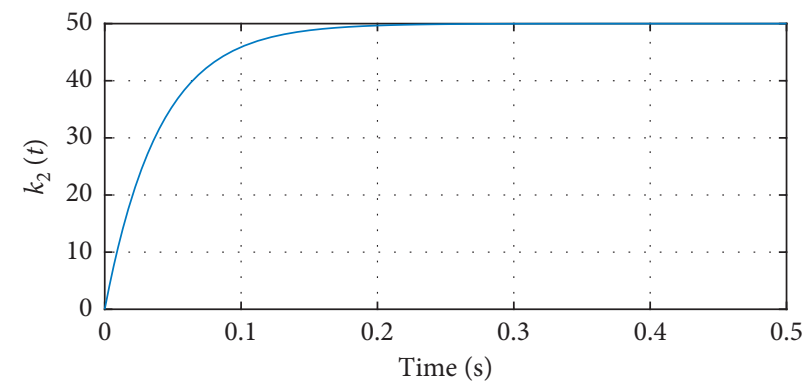

(b)

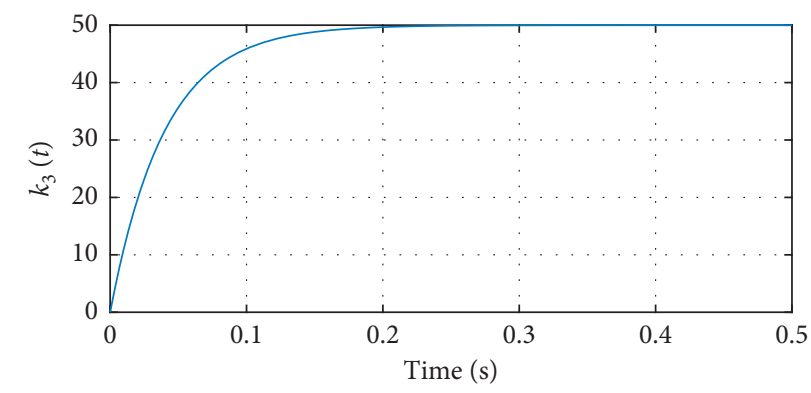

(c)

Figure 5: Observer gain of TVGDO (Case 1).

Figures 6-8 show the simulation results for Case 2. From Figure 6, for DO-based SMC and ESO-based SMC, the undesirable large overshoot of synchronization errors can be observed. And the proposed TVGDO-based SMC can achieve the faster convergence rate than the DO-based and ESO-based schemes. As mentioned before in Section 2.2, the reason is that the spike problem of DO and ESO can be caused by choosing a large observer gain if the initial values of estimate and true states are not equal. Then, the spike output values of observer are transmitted into the control inputs to lead the large overshoot of synchronization errors. The undesirable spike phenomenon of DO and ESO can be observed from Figure 7. Figure 8 shows the undesirable large spike control inputs of DO-based SMC and ESO-based SMC. From Figures 6-8, it is clear that the undesirable spike phenomenon is eliminated in the proposed TVGDO and TVGDO-based SMC. An excellent control performance which is similar to Case 1 still can be guaranteed by the proposed controller and observer. Thus, the spike problem is avoided by the proposed scheme of this study.

According to the simulation results, the following can be concluded:

(1) For conventional ESO and DO, an undesirable spike problem can be caused if the initial values of estimate and true states are not equal. The proposed TVGDO can eliminate the undesirable spike problem (Figures 2-4 and 6-8).

(2) Since the uncertainties have been estimated by proposed TVGDO, the TVGDO-SMC has no 


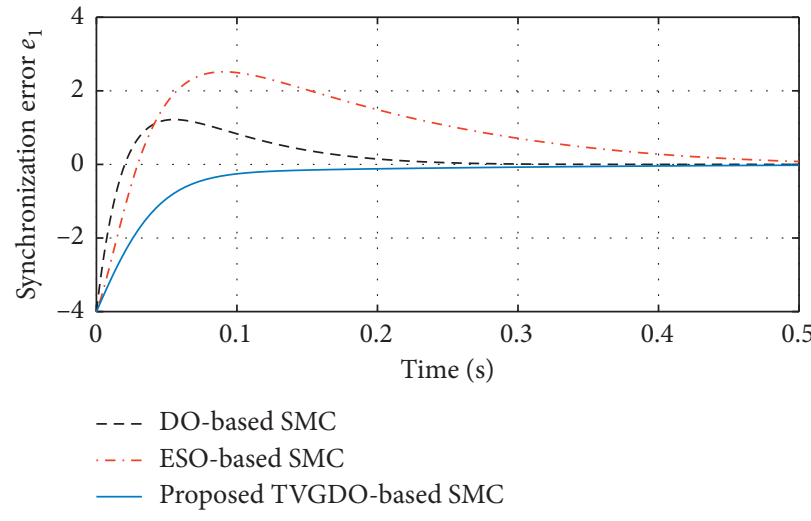

(a)

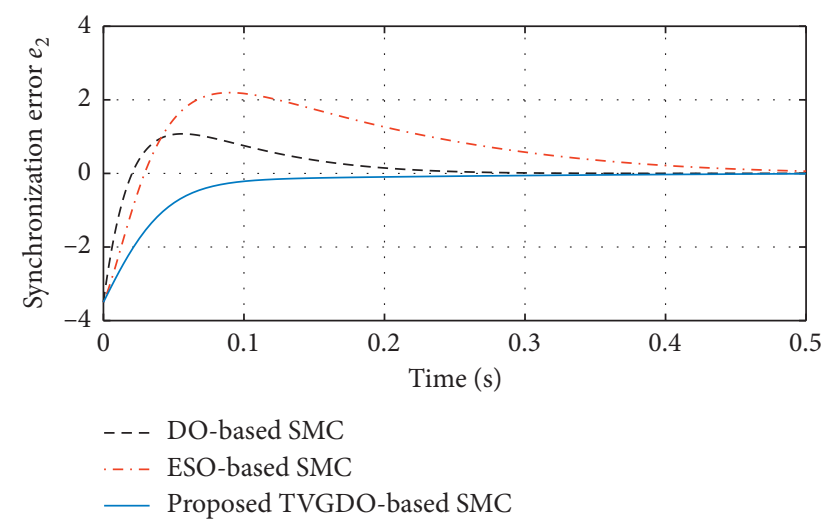

(b)

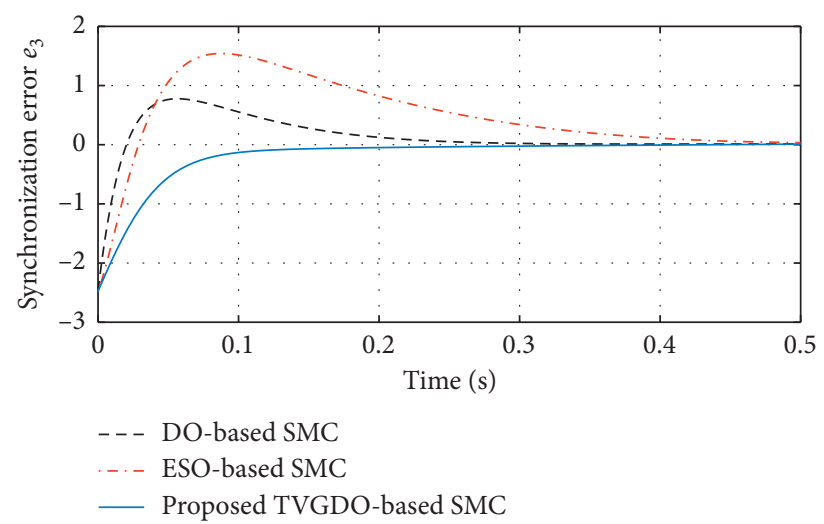

(c)

Figure 6: Synchronization error (Case 2).
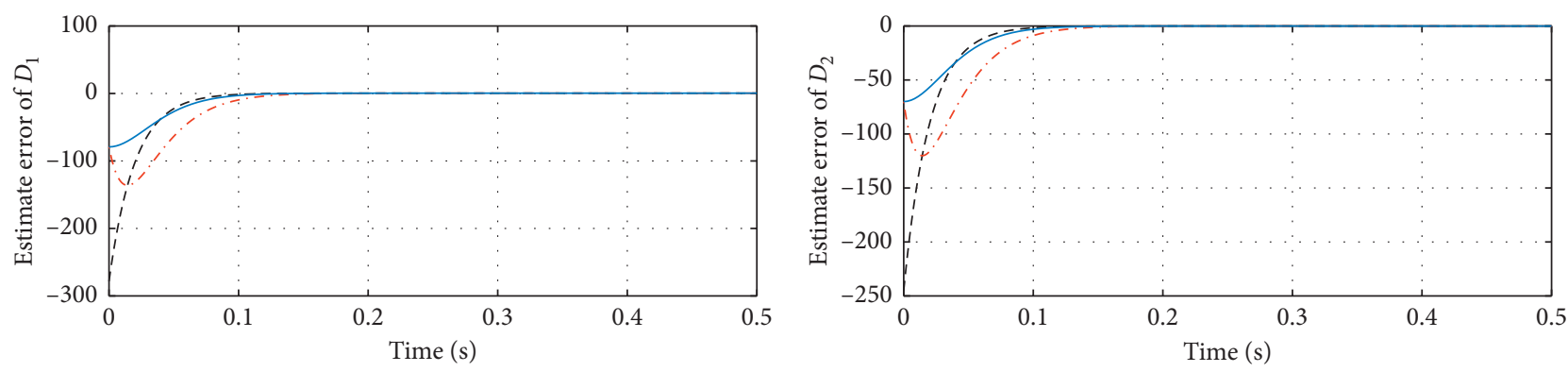

-- DO
-- ESO
- Proposed TVGDO

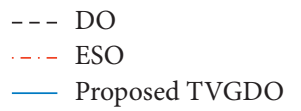

(a)

(b)

Figure 7: Continued. 


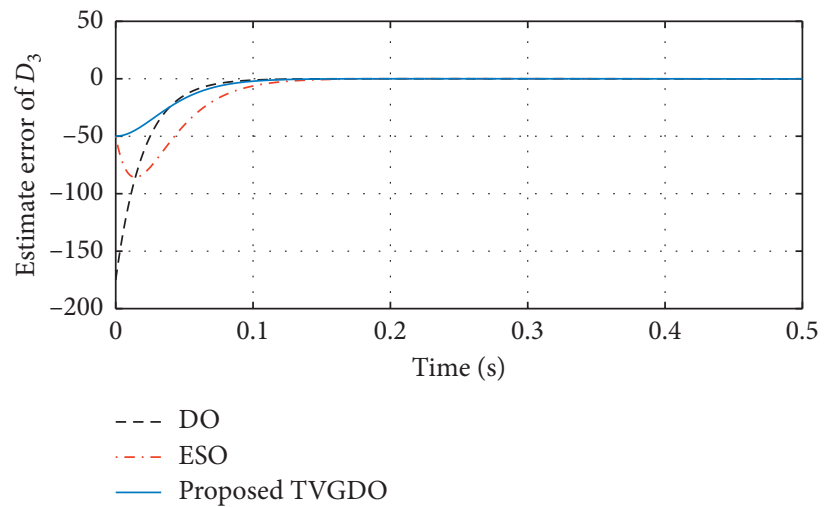

(c)

Figure 7: Estimate error (Case 2).

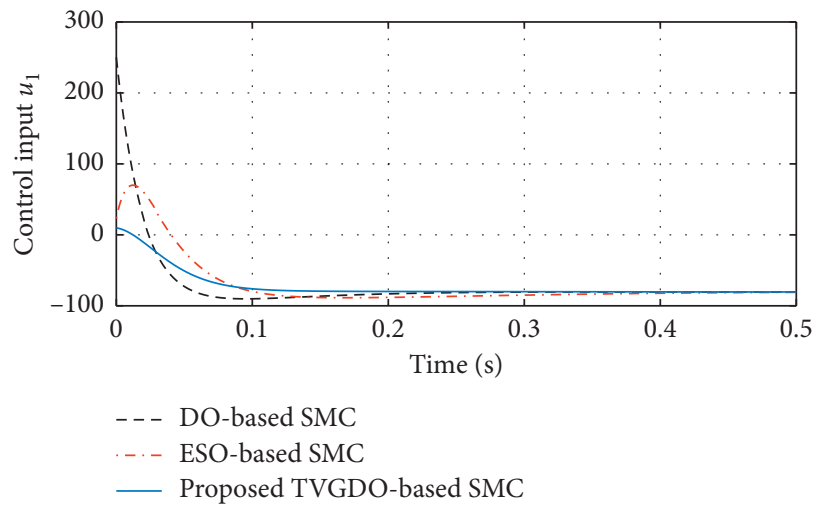

(a)

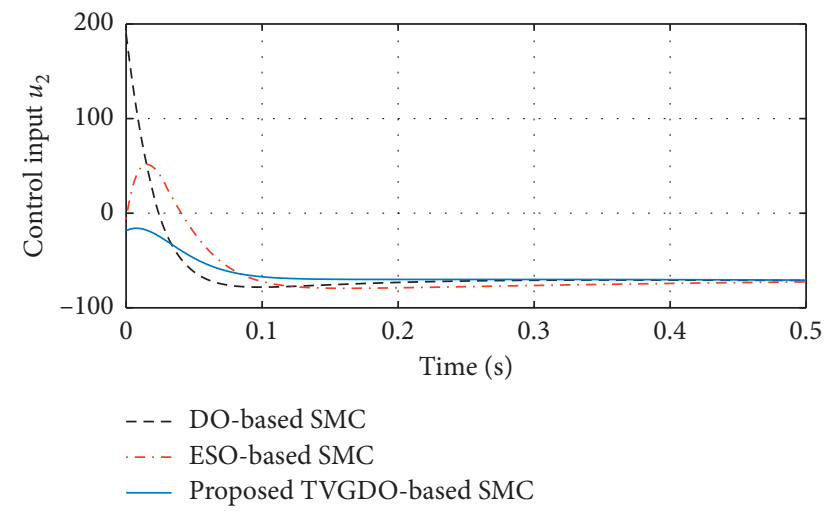

(b)

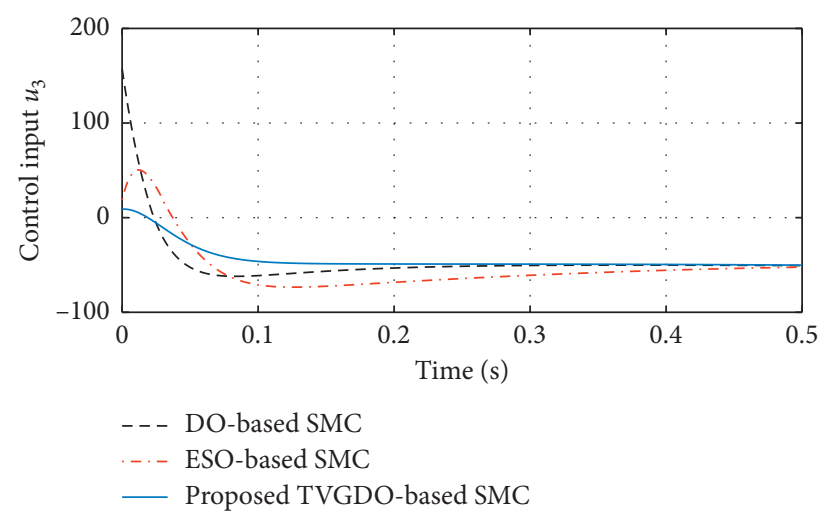

(c)

Figure 8: Control input (Case 2). 
discontinuous control term. Thus, the chattering problem in conventional SMC is solved (Figures 4 and 8). And, unlike the conventional SMC, the proposed controller does not need the upper bound of uncertainties.

\section{Conclusion}

(1) In this study, a novel TVGDO was proposed to estimate the lumped uncertainties and disturbances in the slave chaotic system, which can solve the spike problem in the conventional DO and ESO on the condition of the initial values of estimate and true states are not equal. Moreover, the proposed TVGDO does not need to know the upper bound of uncertainties in advance.

(2) Subsequently, a novel TVGDO-based SMC was proposed to synchronize the chaotic systems. The newly proposed SMC scheme has several advantages over existing SMC. First, the spike problem in the observer-based SMC such as the DO-based and ESObased SMC is solved by the proposed controller. Second, the chattering problem in the conventional SMC also is avoided in the proposed method. Third, unlike the conventional SMC, the proposed method requires no information on the uncertainties.

(3) Finally, mathematical simulation result illustrated the effectiveness of the TVGDO and the proposed TVGDO-based SMC.

\section{Data Availability}

The data used to support the findings of this study are available from the corresponding author upon request.

\section{Conflicts of Interest}

The authors declare that there are no conflicts of interest.

\section{Acknowledgments}

This work was supported by the Natural Science Basic Research Program of Shaanxi (2021JQ-880, 2021JM-533, and 2020JM-646), the Innovation Capability Support Program of Shaanxi (2018GHJD-21), the Science and Technology Program of Xi'an (2019218414GXRC020CG021GXYD20.3), and the Fund of Excellent Doctoral Innovation of Xi'an University of Technology and the Scientific Research Foundation of Xijing University (XJ200202).

\section{References}

[1] E. N. Lorenz, "Deterministic nonperiodic flow," Journal of the Atmospheric Sciences, vol. 20, no. 2, pp. 130-141, 1963.

[2] O. E. Rössler, "An equation for continuous chaos," Physics Letters A, vol. 57, no. 5, pp. 397-398, 1976.

[3] J. Lü, G. Chen, D. Cheng, and S. Celikovsky, "Bridge the gap between the Lorenz system and the chen system," International Journal of Bifurcation and Chaos, vol. 12, no. 12, pp. 2917-2926, 2002.
[4] K. Koubaâ and M. Feki, "Quasi-periodicity, chaos and coexistence in the time delay controlled two-cell DC-DC buck converter," International Journal of Bifurcation and Chaos, vol. 24, no. 10, p. 1450124, 2014.

[5] B. Robert, M. Feki, and H. H. C. Iu, "Control of a pwm inverter using proportional plus extended time-delayed feedback," International Journal of Bifurcation and Chaos, vol. 16, no. 1, pp. 113-128, 2006.

[6] J. Oliveira, P. M. Oliveira, J. Boaventura-Cunha, and T. Pinho, "Chaos-based grey wolf optimizer for higher order sliding mode position control of a robotic manipulator," Nonlinear Dynamics, vol. 90, no. 2, pp. 1353-1362, 2017.

[7] T. Malica, G. Bouchez, D. Wolfersberger, and M. Sciamanna, "Spatiotemporal complexity of chaos in a phase-conjugate feedback laser system," Optics Letters, vol. 45, no. 4, pp. 819-822, 2020.

[8] S. Liu, N. Jiang, A. Zhao, Y. Zhang, and K. Qiu, "Secure optical communication based on cluster chaos synchronization in semiconductor lasers network," IEEE Access, vol. 8, pp. 11872-11879, 2020.

[9] H. Zhang, T. Ma, G. B. Huang et al., "Robust global exponential synchronization of uncertain chaotic delayed neural networks via dual-stage impulsive control," IEEE Transactions on Systems, Man, and Cybernetics, Part B (Cybernetics), vol. 40, no. 3, pp. 831-844, 2009.

[10] V. Vesely, A. Ilka, L. Korosi, and M. Ernek, "Master-slave $\mathrm{H}$-Infinity robust controller design for synchronization of chaotic systems," Modeling, Identification and Control: A Norwegian Research Bulletin, vol. 40, no. 1, pp. 41-50, 2019.

[11] X. Li, Z. Liu, Z. Qiu, Y. Qi, and S. Yuan, "Event-triggered $H \infty$ synchronization control for switched master-slave systems subject to stochastic cyber attacks and quantization," Optimal Control Applications and Methods, vol. 41, no. 2, pp. 690-708, 2020.

[12] X. Chen, J. H. Park, J. Cao, and J. Qiu, “Adaptive synchronization of multiple uncertain coupled chaotic systems via sliding mode control," Neurocomputing, vol. 273, pp. 9-21, 2018.

[13] I. Ahmad and M. Shafiq, "Oscillation free robust adaptive synchronization of chaotic systems with parametric uncertainties," Transactions of the Institute of Measurement and Control, vol. 42, no. 11, pp. 1977-1996, 2020.

[14] Z. Peng, D. Wang, H. Zhang et al., "Distributed neural network control for adaptive synchronization of uncertain dynamical multiagent systems," IEEE Transactions on Neural Networks and Learning Systems, vol. 25, no. 8, pp. 1508-1519, 2013.

[15] C. Mou, C.-s. Jiang, J. Bin, and Q.-x. Wu, "Sliding mode synchronization controller design with neural network for uncertain chaotic systems," Chaos, Solitons \& Fractals, vol. 39, no. 4, pp. 1856-1863, 2009.

[16] R. Sakthivel, R. Sakthivel, O.-M. Kwon, P. Selvaraj, and S. M. Anthoni, "Observer-based robust synchronization of fractional-order multi-weighted complex dynamical networks," Nonlinear Dynamics, vol. 98, no. 2, pp. 1231-1246, 2019.

[17] T. Wang, J. Ge, T. Li et al., "Observer-based $H_{\infty}$ control for synchronization in delayed neural networks under multiple disturbances," International Journal of Control, Automation and Systems, vol. 18, no. 8, pp. 1-12, 2020.

[18] H. Zhang, X.-K. Ma, and W.-Z. Liu, "Synchronization of chaotic systems with parametric uncertainty using active sliding mode control," Chaos, Solitons \& Fractals, vol. 21, no. 5, pp. 1249-1257, 2004. 
[19] S. Vaidyanathan, S. Sampath, and A. T. Azar, "Global chaos synchronisation of identical chaotic systems via novel sliding mode control method and its application to Zhu system," International Journal of Modelling, Identification and Control, vol. 23, no. 1, pp. 92-100, 2015.

[20] A. Zhou and J. Lei, "Research on a novel kind of robust terminal sliding mode synchronization of chaotic systems," Optik, vol. 131, pp. 527-538, 2017.

[21] Y. Gao, J. Ren, and M. Zhao, "Projective lag synchronization of second-order chaotic systems via modified terminal sliding mode control," IMA Journal of Mathematical Control and Information, vol. 34, no. 3, pp. 1045-1059, 2017.

[22] S. Vaidyanathan and A. Rhif, "A novel four-leaf chaotic system, its control and synchronisation via integral sliding mode control," International Journal of Modelling, Identification and Control, vol. 28, no. 1, pp. 28-39, 2017.

[23] S. Lin and W. Zhang, "Chattering reduced sliding mode control for a class of chaotic systems," Nonlinear Dynamics, vol. 93, no. 4, pp. 2273-2282, 2018.

[24] H.-T. Yau and C.-L. Chen, "Chattering-free fuzzy slidingmode control strategy for uncertain chaotic systems," Chaos, Solitons \& Fractals, vol. 30, no. 3, pp. 709-718, 2006.

[25] M. P. Aghababa and M. E. Akbari, "A chattering-free robust adaptive sliding mode controller for synchronization of two different chaotic systems with unknown uncertainties and external disturbances," Applied Mathematics and Computation, vol. 218, no. 9, pp. 5757-5768, 2012.

[26] J. Luo, S. Qu, Z. Xiong, E. Appiagyei, and L. Zhao, "Observerbased finite-time modified projective synchronization of multiple uncertain chaotic systems and applications to secure communication using DNA encoding," IEEE Access, vol. 7, pp. 65527-65543, 2019.

[27] X.-T. Tran and H.-J. Kang, "A novel observer-based finitetime control method for modified function projective synchronization of uncertain chaotic (hyperchaotic) systems," Nonlinear Dynamics, vol. 80, no. 1-2, pp. 905-916, 2015.

[28] D. Qian, H. Ding, S. G. Lee et al., "Suppression of chaotic behaviors in a complex biological system by disturbance observer-based derivative-integral terminal sliding mode," IEEE/CAA Journal of Automatica Sinica, vol. 7, no. 1, pp. 126-135, 2019.

[29] F. Shiravani and M. H. Shafiei, "Robust output regulation via sliding mode control and disturbance observer: application in a forced Van Der Pol chaotic oscillator," Journal of Dynamic Systems, Measurement, and Control, vol. 139, no. 9, 2017.

[30] Y. Zhao, X. Li, and P. Duan, "Observer-based sliding mode control for synchronization of delayed chaotic neural networks with unknown disturbance," Neural Networks, vol. 117, pp. 268-273, 2019.

[31] F. Abu Khadra, "Tracking control of chaotic systems via optimized active disturbance rejection control," Mathematical Problems in Engineering, vol. 2018, Article ID 4698953, 10 pages, 2018.

[32] X. Xi, S. Mobayen, H. Ren, and S. Jafari, "Robust finite-time synchronization of a class of chaotic systems via adaptive global sliding mode control," Journal of Vibration and Control, vol. 24, no. 17, pp. 3842-3854, 2018. 